\title{
Stress Analysis of Gun Barrel Subjected to Dynamic Pressure
}

\author{
H. Babaei*, M. Malakzadeh, H. Asgari \\ Department of Mechanical Engineering, Engineering Faculty, University of Guilan, Rasht, Iran
}

Email address:

ghbabaei@guilan.ac.ir (H. Babaei), hghbabaei@Gmail.com (H. Babaei)

\section{To cite this article:}

H. Babaei, M. Malakzadeh, H. Asgari. Stress Analysis of Gun Barrel Subjected to Dynamic Pressure. International Journal of Mechanical Engineering and Applications. Vol. 3, No. 4, 2015, pp. 71-80. doi: 10.11648/j.ijmea.20150304.14

\begin{abstract}
In the optimal design of a modern gun barrel, there are some aspects to be considered. One of the main factor is internal ballistic which consist of pressure-time, pressure-distance, velocity-time and distance-time curves. In this paper, a simple analytical solution for the plastic stress of an internally pressurized open-ended thick-walled cylinder made of hardening steel which is the closest model to gun barrel is obtained in perfectly plastic and plane stress condition by using energy method and the yield criterion of Von Mises and adding rifle grooves and choosing stress components as basic unknowns and ballistic pressure equation as known. Then results of analytical solution are compared to a numerical model and verified a very well and reliable accuracy. So the resultant can be used easily in calculation of radial expansion velocity and compressive pressure.
\end{abstract}

Keywords: Gun Barrel, Stress, Dynamic Pressure

\section{Introduction}

Many papers are published around the matter of gun design and especially about gun tube design from the beginning of improved guns design generation until now, but reaching to variation sources around this subject is impossible because of preventing of publishing of this technology and security and military problems of some countries. Therefore many engineers and designers who work on this field assume a reasonable simplification that considers gun barrel as an open-ended thick-walled cylinder in which an explosive causes stress and deformation on the wall by creation an immediate pressure. According to this assumption investigation must be done under plane stress condition $\left(\sigma_{z}=0\right)$. Few satisfactory theoretical solution based on Von Mises yield criterion that are reliable and convenient for engineering use have been obtained for an open-ended thick-walled cylinder. A closed-form solution for the stress components was given by Nadai using an auxiliary-variable method and the deformation theory of Hencky [1]. A set of analytical expressions for the elastoplastic stress and displacement components were obtained by Davidson et al. on the basis of an empirical relationship resulting from tests [2]. Two mathematically consistent analytical solutions to the strains and displacements were obtained according to the deformation theory of Hencky and to the flow theory of Prandtl-Reuss, respectively by P.C.T. Chen using a modified Nadai's auxiliary variable method [3]. D.R. Bland et al. derived equations of stress distribution in thick-walled cylinder under internal and external pressure using numerical solution in 1956 [4]. They studied terms of equivalent stress and strain for the two conditions of open-ended $\left(\sigma_{z}=0\right)$ and close-ended $\left(\varepsilon_{z}=0\right)$ for this analysis but the way of calculating of stresses in the open-ended condition which is the appropriate model for gun barrel had not been mentioned. V. A. Adintsov et al. achieved an equation for radial and hoop stresses and radial expansion velocity by analytical investigating on perfectly plastic thick-walled cylinder behavior under internal explosive pressure using energy method [5]. One of the weak points of this study is analyzing the material as a perfectly plastic one but not considering the strain rate. An analytical pattern and some numerical results for an internal-pressurized open-ended thick-walled cylinder made of linear-hardening material was given by $\mathrm{Xu}$ Hong and Chen Shuning on the basis of the Prandtl-Reuss flow theory [6]. A theoretical and numerical analysis for a similar problem was performed by 
LiuYong using the elasto-plastic mixed-boundary-element method [7]. Gao Xin-lin et al. obtained a closed form analytical solution for stress, strain and displacement components for an internal-pressurized elasto-plastic openended thick-walled cylinder in 1991 [8]. In this paper it is shown that the solution is a general one with on the one hand Nadai's known solution for stress components and on the other P.C.T. Chen's solution for strain components of an open-ended thick-walled cylinder made of elastic-perfectlyplastic material as its two specific cases. In this analysis it is assumed an auxiliary function for radial stress in terms of current radius after performing pressure for obtaining stress components at plane stress condition in plastic zone which is very complicated and Time-consuming. X.-L Gao et al. exhibited a technique for elastic-plastic internally pressurized cylinder analyzing in that both Tresca and Von Mises criterion is used for three conditions of plane stress, plane strain and close-ended in order to calculate the stresses and strains in 2003 [9]. Also the deformation energy of cylinder wall is obtained after the pressure is performed. Lack of considering the term of wall expansion acceleration is one of it's weaknesses. Li Mao-lin et al. investigated plastic limit load of viscoplastic thick-walled cylinder and spherical shell subjected to internal pressure analytically using a strain gradient plasticity theory in the paper which is presented in 2008 [10]. Results show that the size effect is more evident with increasing strain or strain rate sensitivity index, but the weak point of this approach is that the viscoplastic model analysis doesn't include material behavior in all part of the wall. Bagheri et al. presented a paper with the purpose of deriving a mathematical model for expansion isotropic thick-walled aluminum cylinder containing TNT in which, JWL equation of state is considered for explosive products [11]. As a result the equations of radial and hoop stress and radial expansion velocity is obtained. However, it must be noted that, each of these known analytical solutions is either oversimplified in the material model but time-consuming in calculation $[12,13]$ or too complicated in the expressions proposed but incomplete in content $[14,15]$.

In this analysis after-explosion phenomena in a thick walled steel cylinder, such as radial pressure on the wall of cylinder, the radial drift, the rate of expansion of the radial drift and radial and circumferential stresses, are considered. This study starts with a simple analytical model using energy-based methods for mentioned parameters. Subsequently, by choosing a cylinder which has specific material and specific geometric dimensions as well as an explosive substance, the mentioned analysis is carried out. One of the advantages of this study is simplicity in calculating the rate of the radial expansion and hence, changes in the radius of the cylinder and its stresses and also the acceleration of the wall are measurable.

\section{Theoretical Analyses}

\subsection{Basic Equations of Thick-Walled Cylinders}

Considering Figure 1, the following equations can be obtained [16]

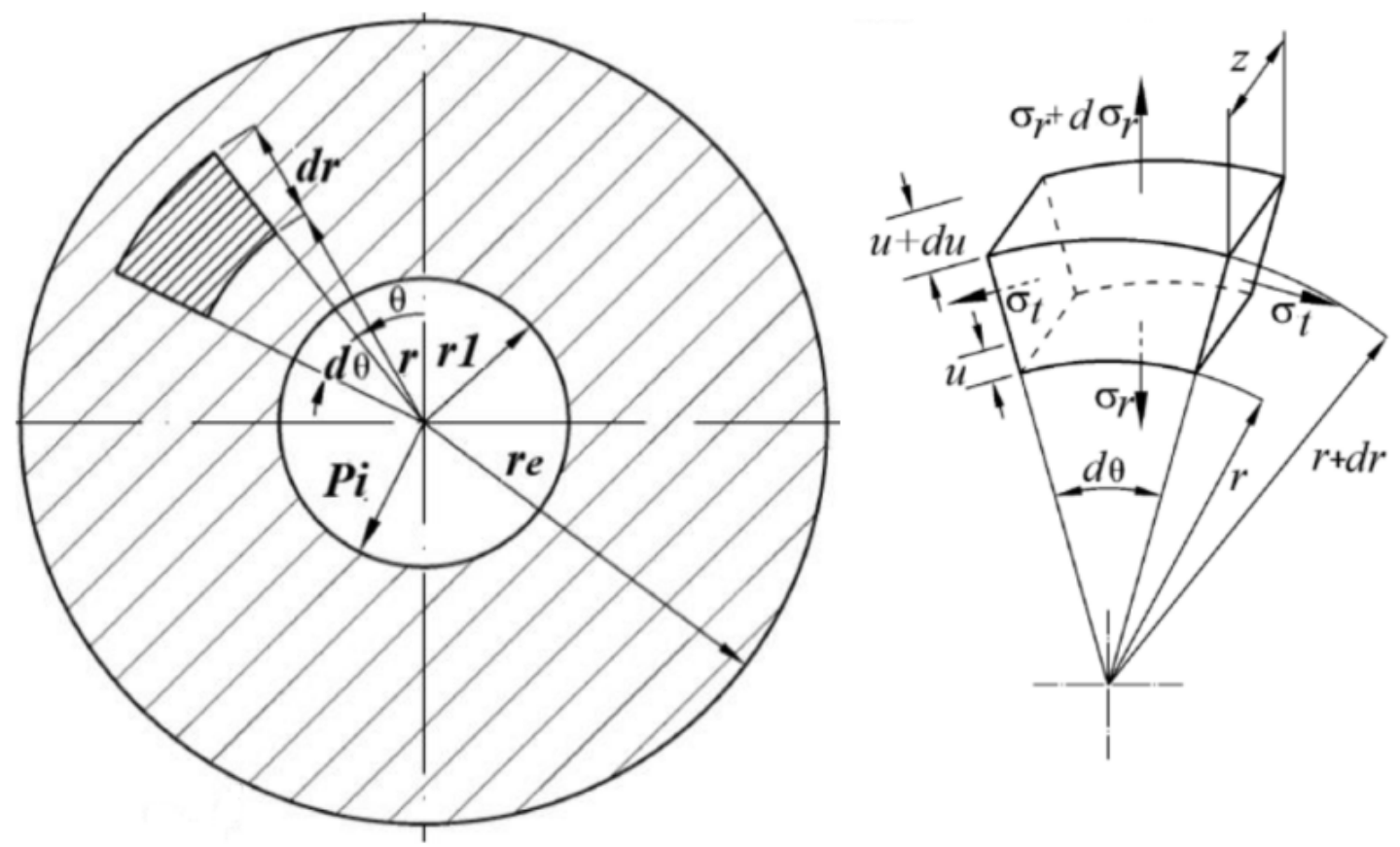

Figure 1. An element of a cylindrical body and its related components.

$$
\frac{d \sigma_{r}}{d r}+\frac{\sigma_{r}-\sigma_{\theta}}{r}=0
$$

Where $\sigma_{r}$ and $\sigma_{\theta}$ are radial and hoop stresses respectively. The equation above shows the equation of the thick-walled cylinder in static state [17] and considering the open-ended condition $\left(\sigma_{z}=0\right)$, and in a dynamic state it turns to the following equation considering the movement of the element shown in figure 1 . It should be mentioned that the term 
related to density in acceleration is added to the equation [18].

$$
\frac{d \sigma_{r}}{d r}+\frac{\sigma_{r}-\sigma_{\theta}}{r}=\rho \frac{d v_{r}}{d t}=\rho \dot{v}_{r}
$$

Where $v_{r}$ and $\dot{v}_{r}$ are radial expansion velocity and acceleration respectively, and $\rho$ is the density of cylinder material.

\subsection{Conservation Law}

Conservation law in physics expresses that the whole energy of an isolated system is consistent with time [19]. Energy neither produces nor wastes, but transforms from a state to another. In this study the chemical energy of explosives turns to kinetic energy. this law is explained in the form of the equation below.

$$
E+W+E_{f}+W_{r}=E_{0}
$$

where, $E_{0}$ and $E$ represent initial internal energy and current energy of the substances of explosion, respectively. $W$ is the kinetic energy of the wall of the cylinder, $E_{f}$ is the work of plastic deformation and $\mathrm{W}_{\mathrm{r}}$ is the work of friction force of the rifle. If we represent the equation above per unit of length, will have the following equation.

$$
\bar{E}+\bar{W}+\bar{E}_{f}+\bar{W}_{r}=1
$$

The terms of the equation above are determined during the trend of analysis in this study.

\subsection{Adiabatic Expansion Law}

In this research the expansion of the wall due to explosion is considered adiabatic and obeys the equation below [20].

$$
p V^{\gamma}=\text { const }
$$

Where $p$ and $V$ is pressure and special volume respectively and $\gamma$ is adiabatic expansion coefficient which is specified for any material.

\subsection{Gunnery Internal Ballistic Equations}

Internal ballistic equations consist of formulas and diagrams related to time-pressure, distance-pressure and distance-time. It should be mentioned that, because the selected weapon in this study is M24 which has a $500 \mathrm{~mm}$ barrel and the firing time is $1 \mathrm{~ms}$, we consider the pressure curve yielded from Russel Model within these ranges and subsequently, we curve fit this diagram in software. The results are as follows.

The equation of pressure based on time:

$$
P=a_{1} e^{-\left(\frac{t-b_{1}}{c_{1}}\right)^{2}}+a_{2} e^{-\left(\frac{t-b_{2}}{c_{2}}\right)^{2}}+a_{3} e^{-\left(\frac{t-b_{3}}{c_{3}}\right)^{2}}
$$

where $P$ represents internal ballistic pressure and $t$ represents the time interval between triggering and firing bullet through the barrel.

$$
\begin{gathered}
a_{1}=2.287 \times 10^{8}, b_{1}=0.000358, c_{1}=0.000147, \\
a_{2}=7.827 \times 10^{7}, b_{2}=0.0004937, c_{2}=0.000350, \\
a_{3}=1.999 \times 10^{8}, b_{3}=0.0004937, c_{3}=0.0002388
\end{gathered}
$$

Hence, figure 2 shows the diagram of equation (5)

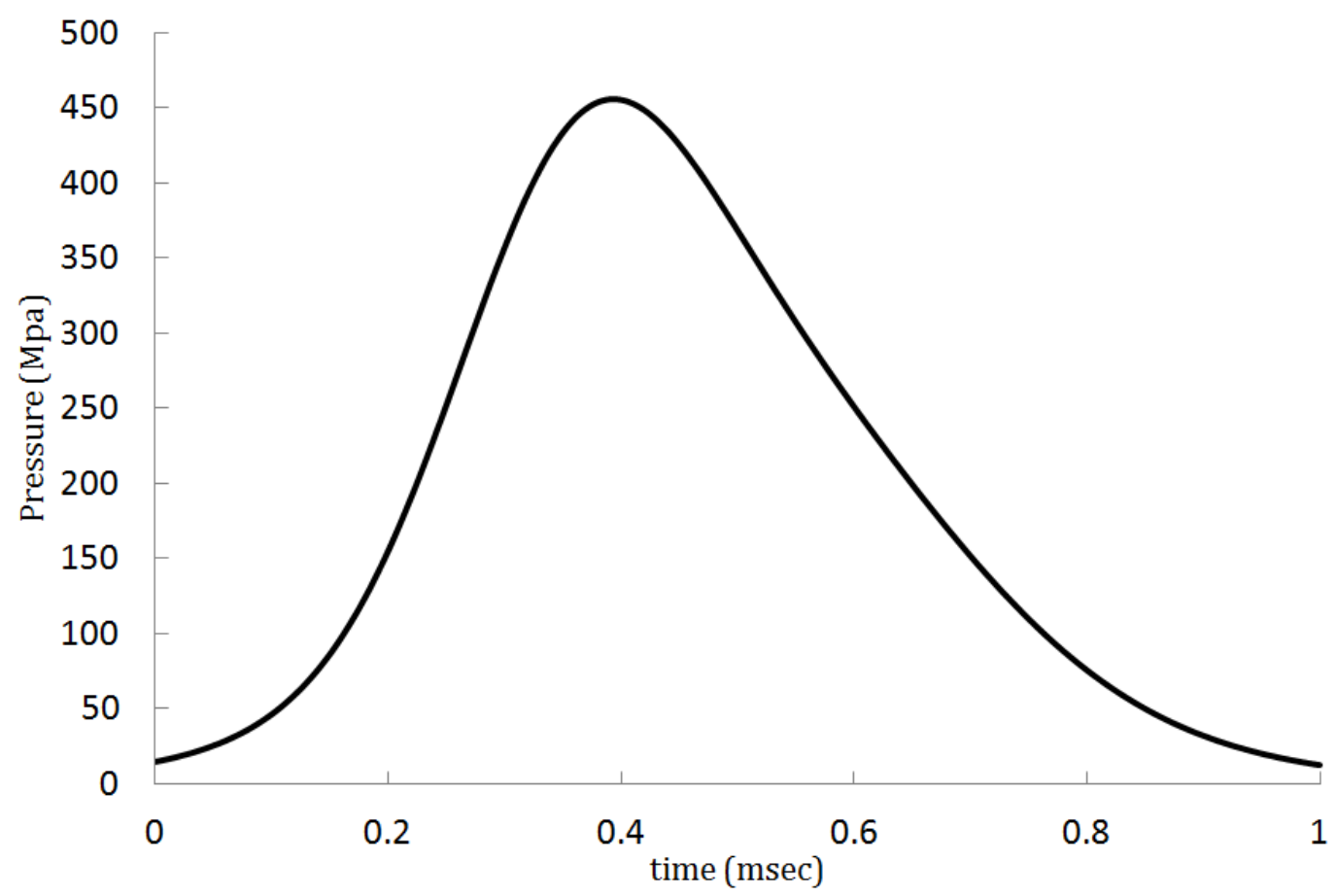

Figure 2. the internal ballistic pressure versus triggering time.

The equation of distance (the amount of space passed by the bullet into the barrel) based on time is presented as 
follows.

$$
m=-0.01431, n=9424, q=23.44, s=3384
$$

$$
x=m e^{n \times t}+q e^{s \times t}-m-q
$$

The yielded diagram from the equation above is demonstrated in the Figure 3.

In which $x$ is the length of the barrel

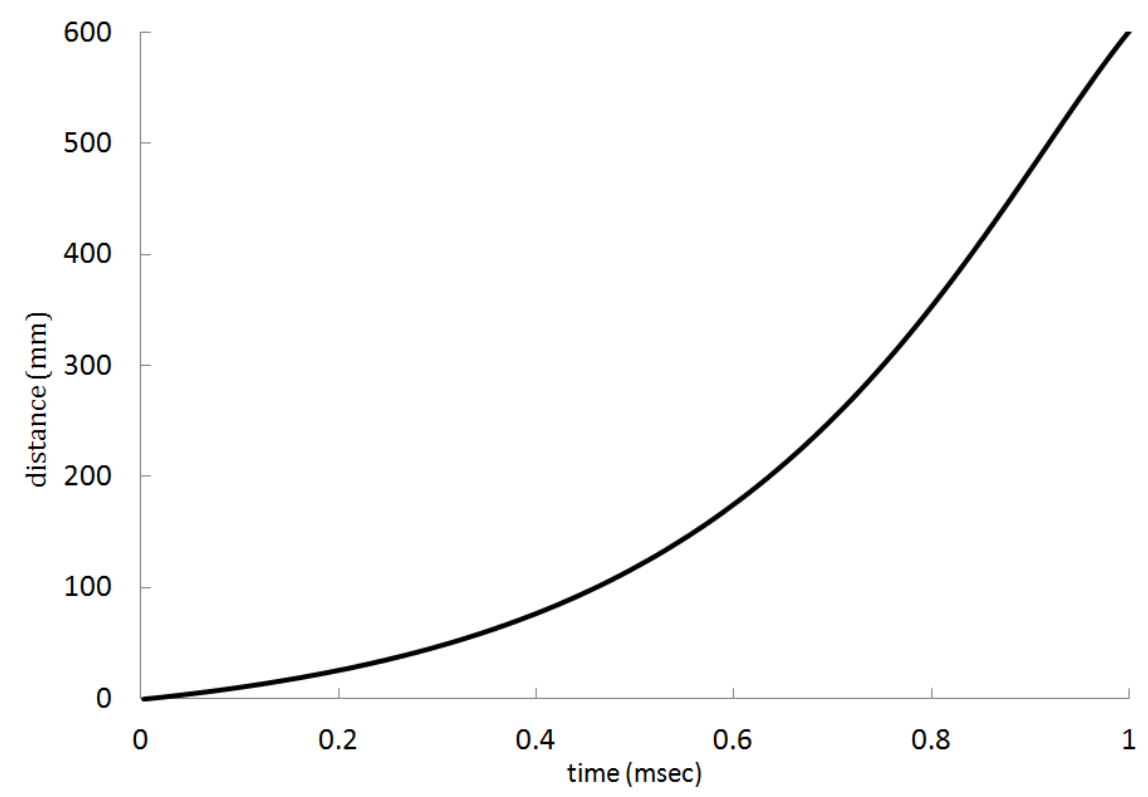

Figure 3. the distance passed by the bullet into the barrel against time.

As a result, the diagram of pressure versus distance is demonstrated as Figure 4.

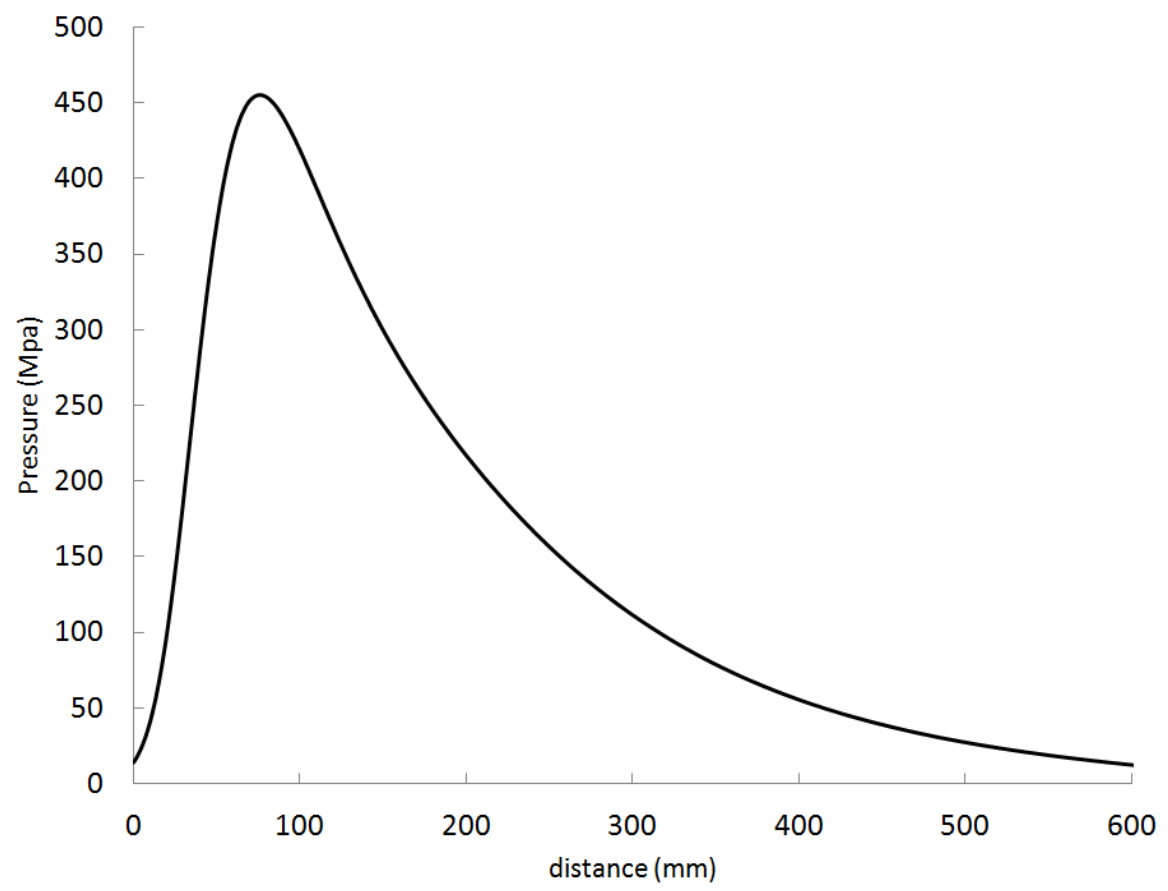

Figure 4. inside ballistic pressure versus distance.

\section{Research Method}

\subsection{Assumptions}

- In this analysis the cylinder containing explosives is assumed perfectly plastic, homogeny and incompressible.

- The used criterion is Von Mises. As a result of recent studies and comparing with the analysis a more appropriate criterion is introduced which is Von Mises criterion. Due to utilizing plastic work, the simulation and the analysis are independent of stiffness strains and 
thermal variations.

- The volume of the barrel is assumed constant before and after explosion and there is no body force affecting the barrel but the pressure of explosion.

- In this analysis the shock wave loading and swinging effects on the diameter of the barrel are ignored.

- The barrel analysis is carried out under the conditions of plane stresses $\left(\sigma_{z}=0\right)$.

- It is assumed that axial stresses of the barrel caused by the friction force of the projectile do not affect the analysis results.

- In the equation of conservation law, the term related to the energy of explosion is neglected.

\subsection{Analysis}

The stresses $\sigma_{r}$ and $\sigma_{\theta}$ are principal. The internal and external initial cylinder radii before the deformation due to explosion are denoted by $a_{0}$ and $b_{0}$ respectively and radii after deformation by $a$ and $b$, and $\mathrm{r}$ is the current radii. We assume the deformation of the tube wall is perfectly plastic, so there is no changing in volume, therefore from equality of current and initial volume we have:

$$
\sigma_{r}=\left(\alpha \sigma_{y}+\rho v_{a}^{2}+\rho a \dot{v}_{a}\right) \ln \frac{r}{a}+\frac{1}{2} \rho v_{a}^{2}\left(\frac{a^{2}}{r^{2}}-1\right)-p(x, t)
$$

Where $p(x, t)$ is the pressure dependent to distance and time that is considered as $p$ from now on. We have $\sigma_{r}=0$ in $r=b$, the equation of internal radii acceleration is as follow:

$$
\dot{v}_{a}=\frac{p}{\rho a \ln \frac{b}{a}}-\frac{\alpha \sigma_{y}}{\rho a}-v_{a}^{2}\left(\frac{1}{a}+\frac{a^{2}-b^{2}}{2 a b^{2} \ln \frac{b}{a}}\right)
$$

Now we want to calculate the amount of $v_{a}$ and $\dot{v}_{a}$. Accordingly, in order to preventing of using complicated calculation of differential equation, we present a simple solution using energy method (conservation energy law) and thermodynamic fundamental. Hence we calculate each term of equation (3).

\subsubsection{Calculation of Detonation Products Internal Energy Per Unit of Length $(\bar{E})$}

$p_{0}$ is instantaneous pressure and obtained from

$$
p_{0}=\frac{\rho_{0} D^{2}}{8}
$$

$\rho_{0}$ is the density of the explosive and $\mathrm{D}$ is detonation velocity which are specified for any explosive and obtained empirically and accessible in engineering handbooks.

Internal energy for an ideal gas is in form $E=\frac{p V}{\gamma-1}$ in which $V=\pi a^{2}$ is volume per unit of length, so we have

$$
\bar{E}=\frac{E}{E_{0}}=\frac{p}{p_{0}}\left(\frac{a}{a_{2}}\right)^{2}
$$

\subsubsection{Calculation of Wall Kinetic Energy Per Unit of \\ Length $(\bar{W})$}

The equation of wall kinetic energy is in form $W=$ $\int_{a}^{b} \frac{1}{2} v_{r}^{2} d m$, substituting (8) and $d m=2 \pi \rho r d r$ in the

$$
r^{2}-a^{2}=r_{0}^{2}-a_{0}^{2}
$$

By substituting $b$ in (5) and differentiating, $v_{a}$ (internal radius expansion velocity) and $v_{r}$ (current radius expansion velocity) is obtained, so [21]:

$$
v_{r}=\frac{a}{r} v_{a}
$$

By differentiating form (6) and substituting in (2) we have

$$
\frac{d \sigma_{r}}{d r}+\frac{\sigma_{r}-\sigma_{\theta}}{r}=\rho\left(\frac{v_{a}^{2}+a \dot{v}_{a}}{r}-\frac{a v_{a}^{2}}{r^{3}}\right)
$$

The yield criterion adopted here is the Von Mises criterion according to recent studies which say it is more accurate than others like Tresca, therefore

$$
\sigma_{\theta}-\sigma_{r}=\alpha \sigma_{y}
$$

Where $\alpha=\frac{2}{\sqrt{3}}$ for Von Mises criterion and $\sigma_{y}$ is yield stress. By integrating (7) and applying the boundary condition $\sigma_{r}=-p$ in $r=a$ :

equation, it turns to $W=\pi \rho a^{2} v_{a}^{2} \ln \left(\frac{b}{a}\right)$, in which $\rho$ is barrel material density, so

$$
\bar{W}=\frac{W}{E_{0}}=\frac{(\gamma-1) \rho v_{a}^{2}\left(\frac{a_{0}}{a}\right)^{2} \ln \left(\frac{b}{a}\right)}{p_{0}}
$$

\subsubsection{Calculation of Work of Plastic Deformation Per Unit of Length $\left(\bar{E}_{f}\right)$}

Work of plastic deformation equation is $E_{f}=$ $\sqrt{3} \alpha \sigma_{y} \pi \int_{a}^{b} \varepsilon_{i} r d r$, where $\varepsilon_{i}$ is equivalent strain, We assume $E^{\prime}=\int_{a}^{b} \varepsilon_{i} r d r$, for solving the integral we need to obtain an equation for the equivalent strain in plane stress condition [4]

$$
\varepsilon_{i}^{p}=\frac{2}{\sqrt{3}}\left(\frac{c}{r^{2}}-\frac{1-v^{2}}{E}\left(\alpha \sigma_{y}+\rho r \dot{v}_{r}\right)\right)
$$

Where $C=\frac{1-v^{2}}{E}\left(\alpha \sigma_{y} r_{c}^{2}\right)$, in which $r_{c}$ is the boundary of elastic and plastic domain and $v$ is poisson coefficient. Since the workplace of this research is perfectly plastic, we have $r_{c}=b$, as a result the equation of equivalent is

$$
\varepsilon_{i}=\frac{2}{\sqrt{3}} \frac{1-v^{2}}{E}\left(\frac{\alpha \sigma_{y} b^{2}}{r^{2}}-\left(\alpha \sigma_{y}+\rho r \dot{v}_{r}\right)\right)
$$

In order to obtain the amount of $\dot{v}_{r}$ we differentiate from (8), therefore

$$
\dot{v}_{r}=\frac{v_{a}^{2}+a \dot{v}_{a}}{r}-\frac{a v_{a}^{2}}{r^{3}}
$$

Substituting (12) and (18) into (17) 


$$
\varepsilon_{i}=\frac{2}{\sqrt{3}} \frac{1-v^{2}}{E}\left(\alpha \sigma_{y}\left(\frac{b^{2}}{r^{2}}-1\right)+\rho\left(\frac{a^{2} v_{a}^{2}}{r^{2}}-\frac{p}{\rho \ln \frac{b}{a}}+\frac{\alpha \sigma_{y}}{\rho}+\frac{\left(a^{2}-b^{2}\right) v_{a}^{2}}{2 b^{2} \ln \frac{b}{a}}\right)\right)
$$

As a result

$$
E^{\prime}=\int_{a}^{b} \varepsilon_{i} r d r=\frac{2}{\sqrt{3}} \frac{1-v^{2}}{E}\left[\alpha \sigma_{y}\left(b^{2} \ln \frac{b}{a}\right)+v_{a}^{2} \rho\left(a^{2} \ln \frac{b}{a}+\frac{\left(a^{2}-b^{2}\right)\left(b^{2}-a^{2}\right)}{4 b^{2} \ln \frac{b}{a}}\right)-\frac{p\left(b^{2}-a^{2}\right)}{2 \ln \frac{b}{a}}\right]
$$

Finally work of plastic deformation equation is in form

$$
\bar{E}_{f}=\frac{E_{f}}{E_{0}}=\frac{\sqrt{3} \alpha \sigma_{y}(\gamma-1)}{p_{0} a_{0}^{2}} E^{\prime}
$$

\subsubsection{Calculation of Work of Rifiling Friction Force Per Unit of Length $\left(\bar{W}_{r}\right)$}

According to number of grooves the equation $F=P A$ can be in form $F(x, t)=P(x, t)\left(\frac{\pi}{4} D^{2}+n h b_{g}\right)$, in which $P(x, t)$ is gas pressure in the place $\mathrm{x}$ and time $\mathrm{t}, F(x, t)$ is gas force on the chamber basis in the place $x$ and time $t, D$ is the caliber, $\mathrm{n}$ is the number of grooves, $\mathrm{h}$ is the grooves depth, and $b_{g}$ is land width.

On the other hand work of friction force is

$$
W_{r}=\mu_{0}\left(\frac{2 i}{d}\right)^{2} \tan \alpha \int_{0}^{L} F(x, t) d x
$$

Where $\mu_{0}$ is average friction coefficient, $\frac{2 i}{d}$ is of physical properties of bullet that is 0.74 on average, $\alpha$ is groove angle and equal to 8 degree according to compare variation of guns. So $\tan \alpha=0.15$. we choose the barrel length $500 \mathrm{~mm}$. finally rifle work of friction force per unit length is

$$
\bar{W}_{r}=\frac{W_{r}}{E_{0}}=\frac{73.26 \mu_{0} F(x, t)(\gamma-1)}{\pi p_{0} a_{0}^{2}}
$$

Substituting equations

$$
\begin{aligned}
& \frac{p}{p_{0}}\left(\frac{a}{a_{2}}\right)^{2}+\frac{(\gamma-1) \rho\left(\frac{a_{0}}{a}\right)^{2} \ln \left(\frac{b}{a}\right)}{p_{0}} v_{a}^{2}+\frac{2 \alpha \sigma_{y(\gamma-1)\left(1-v^{2}\right)}}{E p_{0} a_{0}^{2}}\left[\alpha \sigma_{y} b^{2} \ln \frac{b}{a}+\left(\rho a^{2} \ln \frac{b}{a}+\frac{\rho\left(a^{2}-b^{2}\right)\left(b^{2}-a^{2}\right)}{4 b^{2} \ln \frac{b}{a}}\right) v_{a}^{2}-\frac{p\left(b^{2}-a^{2}\right)}{2 \ln \frac{b}{a}}\right] \\
& +\frac{73.26 \mu_{0} F(x, t)(\gamma-1)}{\pi p_{0} a_{0}^{2}}=1
\end{aligned}
$$

For simplification of recent equation, we assume $A=$ $\frac{p}{p_{0}}\left(\frac{a}{a_{2}}\right)^{2}, B=\frac{(\gamma-1) \rho\left(\frac{a_{0}}{a}\right)^{2} \ln \left(\frac{b}{a}\right)}{p_{0}}, C=\frac{2 \alpha \sigma(\gamma-1)\left(1-v^{2}\right)}{E p_{0} a_{0}^{2}}, D=$ $\alpha \sigma_{y} b^{2} \ln \frac{b}{a}, F=\rho a^{2} \ln \frac{b}{a}+\frac{\rho\left(a^{2}-b^{2}\right)\left(b^{2}-a^{2}\right)}{4 b^{2} \ln \frac{b}{a}}, G=\frac{p\left(b^{2}-a^{2}\right)}{2 \ln \frac{b}{a}}$, and $H=\frac{73.26 \mu_{0} F(x, t)(\gamma-1)}{\pi p_{0} a_{0}^{2}}$

So $v_{a}$ is obtained as

$$
v_{a}=\sqrt{\frac{1}{B+C F}(1-A-C D+C G-H)}
$$

Now by substituting $v_{a}$ into (12), (11) and (10) we obtain $\dot{v}_{a}, \sigma_{r}$ and $\sigma_{\theta}$ respectively.

\subsection{Numerical Simulation}

In this research ABAQUS 6.12 and dynamic explicit solver is used to simulate and solve the problem.

CATIA software is used to model the M24 barrel. The barrel have four grooves with the pitch of a round per twelve inches in accordance with Figure 5.

This model imported to ABAQUS to numerical analysis. Other assumptions and geometrical and physical properties of material and explosive properties is shown in table 1 and table2 respectively.
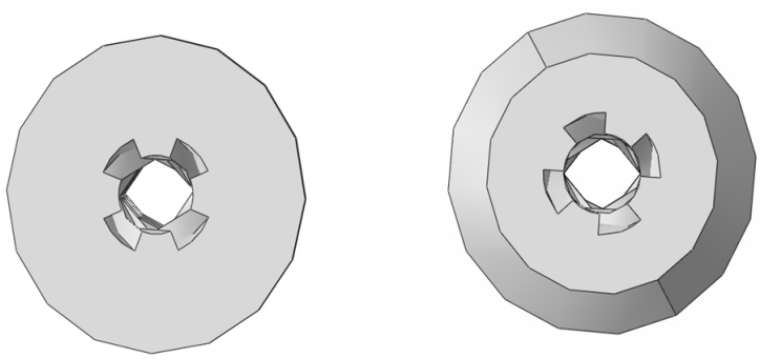

Figure 5. behind and front of barrel with 4 grooved.

Table 1. stainless steel $416 R$ tube properties of M24 model gun used in analysis and simulation.

\begin{tabular}{ll}
\hline Dimension and size & \\
\hline internal radius $(\mathrm{mm})$ & 3.81 \\
External radius $(\mathrm{mm})$ & 14.6 \\
Final expansion radius $(\mathrm{mm})$ & 14 \\
Barrel length $(\mathrm{mm})$ & 600 \\
Groove width $(\mathrm{mm})$ & 3 \\
Groove depth $(\mathrm{mm})$ & 2 \\
Number of rifles & 4 \\
Physical properties & \\
Density $\left(\mathrm{kg} / \mathrm{m}^{3}\right)$ & 7800 \\
Yield stress $(\mathrm{Mpa})$ & 330 \\
Poisson coefficient & 0.3 \\
\hline
\end{tabular}


Table 2. explosive properties [22].

\begin{tabular}{ll}
\hline Density $\left(\mathrm{kg} / \mathrm{m}^{3}\right)$ & 1717 \\
Detonation velocity $(\mathrm{m} / \mathrm{s})$ & 7980 \\
Gas coefficient $\gamma$ & 2.7 \\
\hline
\end{tabular}

The equation of pressure-distance imported in analytical field section to investigate pressure effect. The initial part of barrel is bounded to create boundary condition.

According to the analytical solution, period of $0.001 \mathrm{sec}$ is chosen to numerical analysis in step section in ABAQUS. Mesh section is used for modeling and reticulation of problem geometry. The model have free reticulation and the elements are quadratic. Form of meshing used in barrel is shown as Figure 6.

Solving the problem is done by job section. Other setting related to problem solution in parallel processing condition, output numbers accuracy, etc can be done in this section. Stress contour among barrel length is shown in Figure 7.

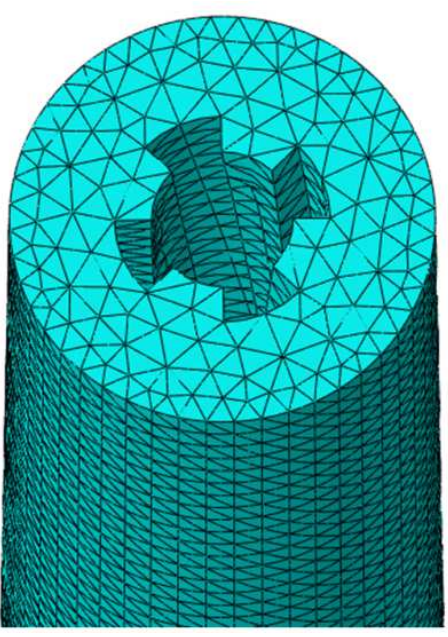

Figure 6. form of meshed barrel.
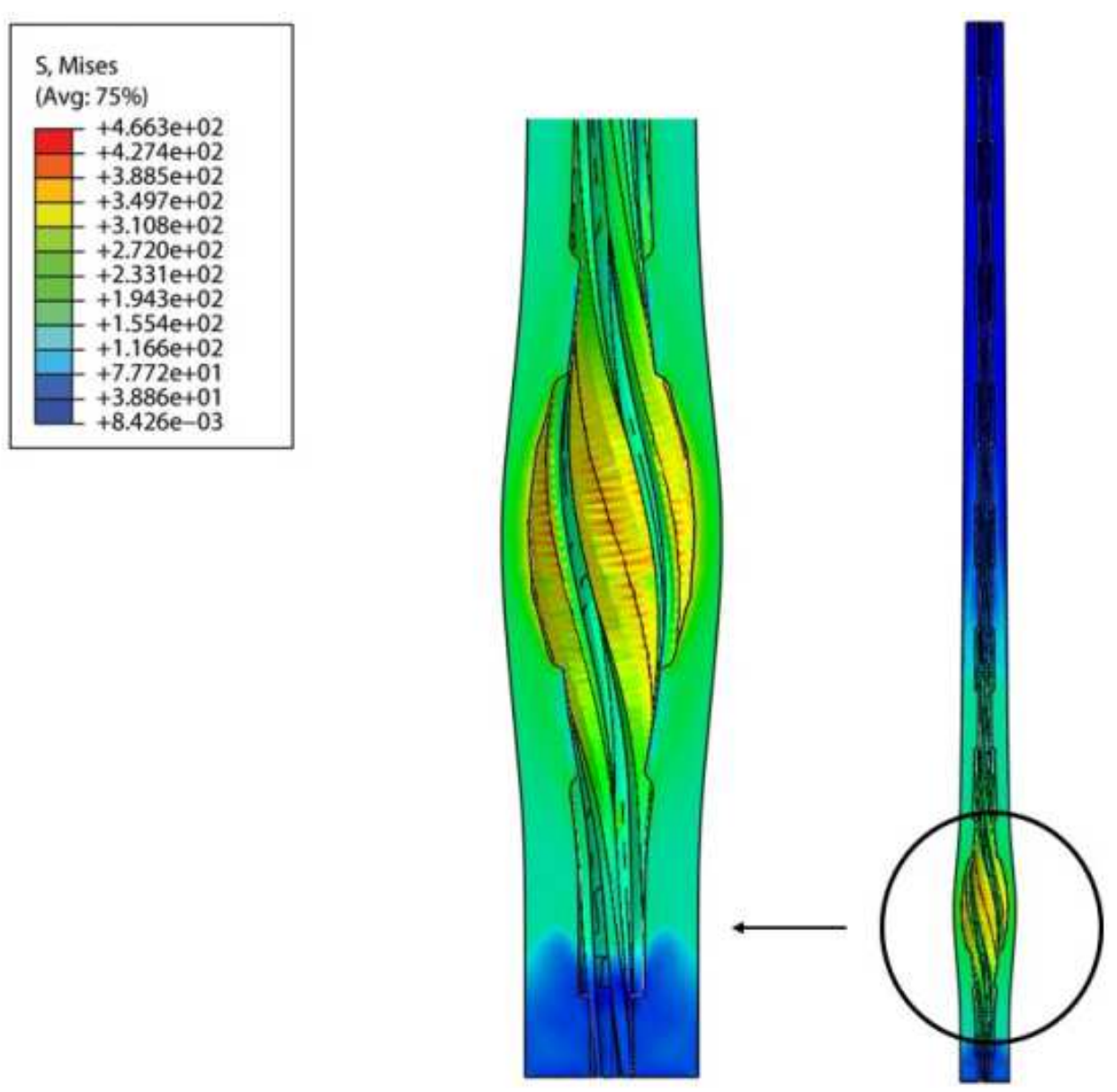

Figure 7. Stress contour among barrel length.

Graph of stress distribution on grooves among of barrel length is presented in Figure 8. 


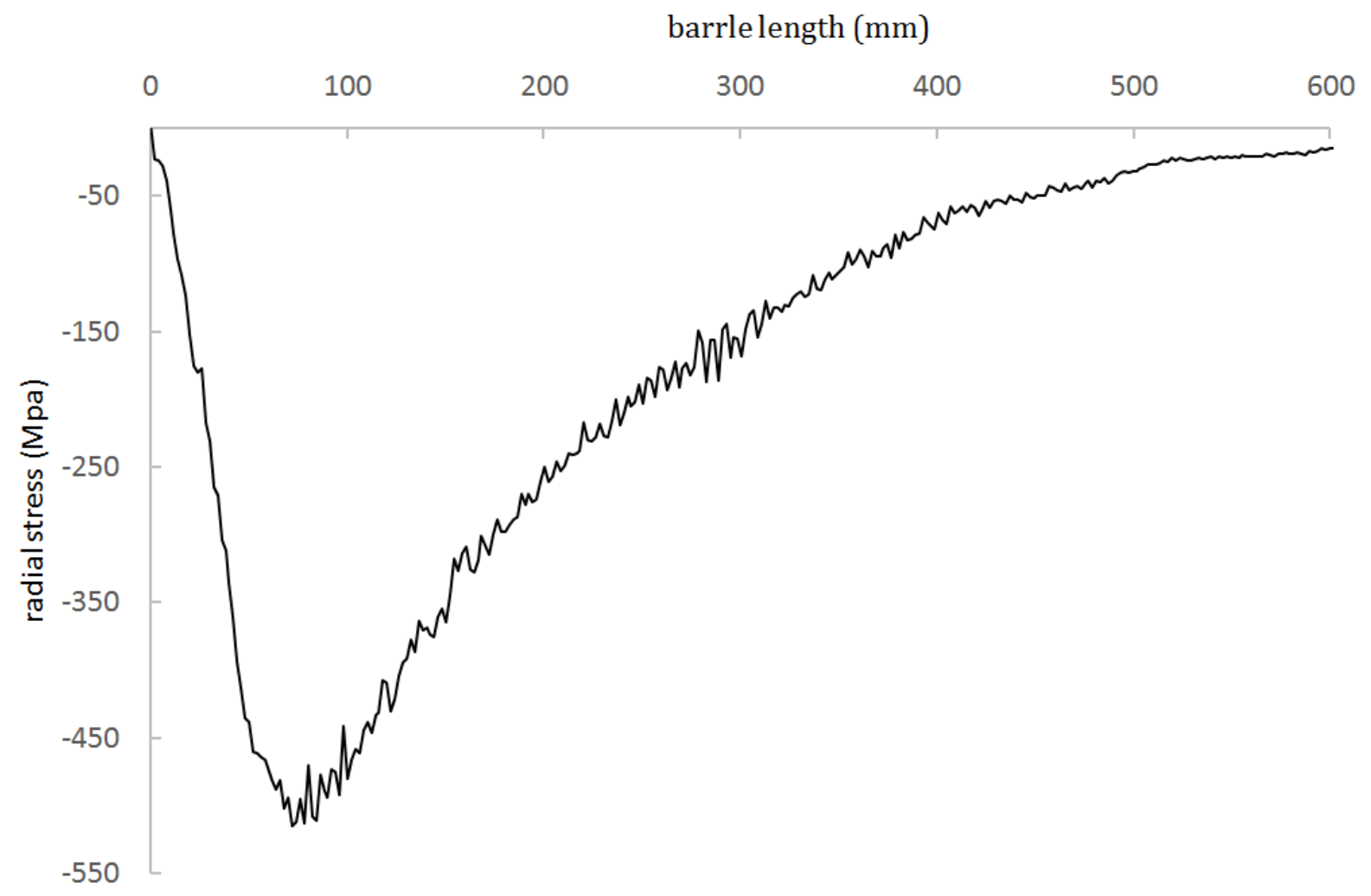

Figure 8. Graph of stress distribution per unit of length.

\section{Conclusion}

Diagram of radial expansion velocity variation per time is shown in Figureure 9 as one of the important results of this research. Maximum tube expansion velocity is $722.04 \mathrm{~m} / \mathrm{s}$ in
$0.39 \mathrm{sec}$ for 4 rifiled barrel and $709.02 \mathrm{~m} / \mathrm{s}$ in same time for 3 rifiled barrel on internal surface. The tube has maximum deformation and minimum thickness in this time. So the more number of grooves, the more expansion velocity.

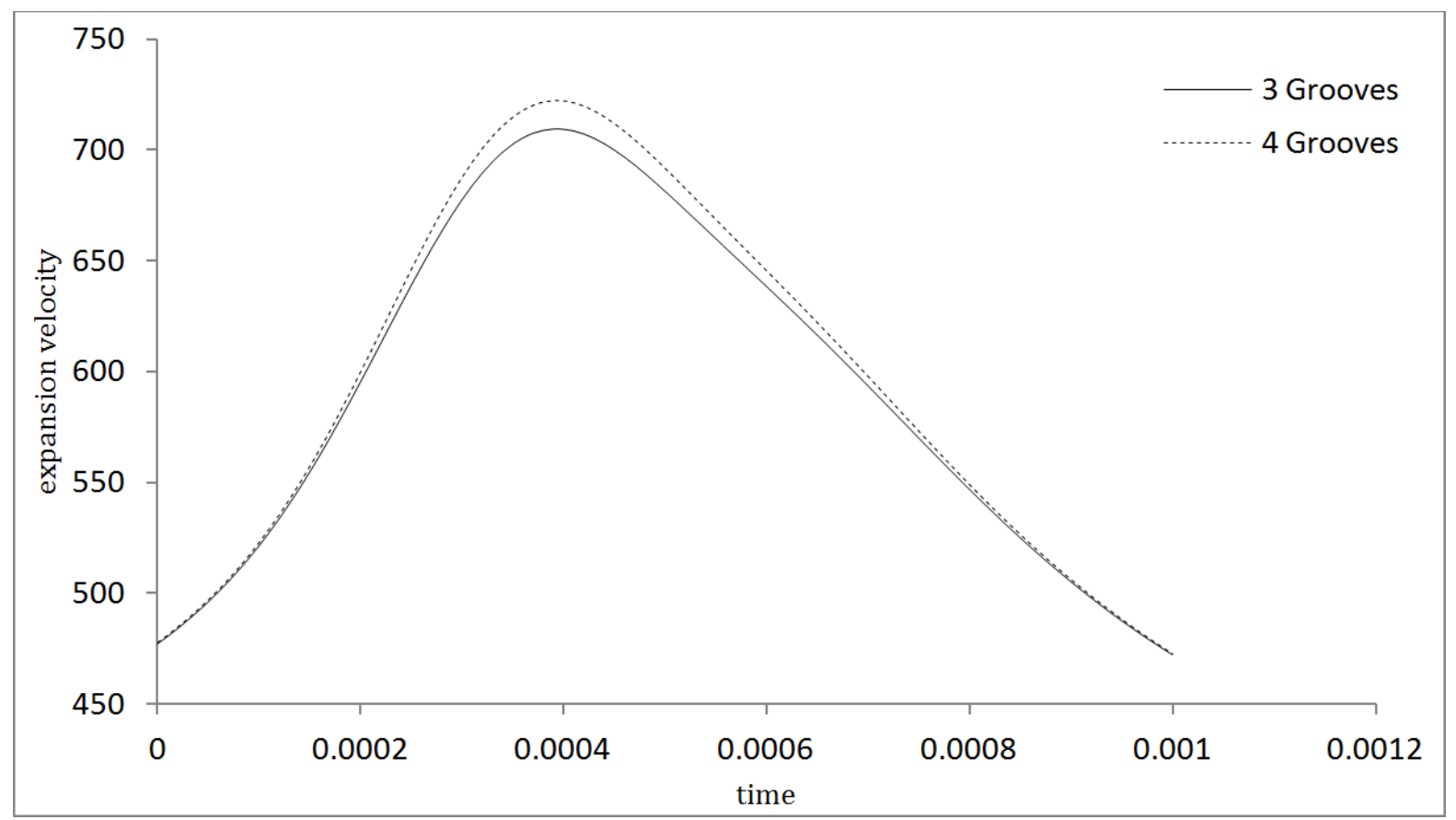

Figure 9. graph of radial expansion velocity variation per time.

Figureure 10 shows comparison of stress diagram per barrel length between two condition of analytical and 
numerical solution on internal surface. Maximum compressive pressure is about $455 \mathrm{Mpa}$ due to analytical solution and about $465 \mathrm{Mpa}$ due to numerical one. As a result the equations obtained from theoretical analysis are in well accordance on numerical solution for calculating the amounts of compressive pressure in order to acceptable difference between the two graphs.

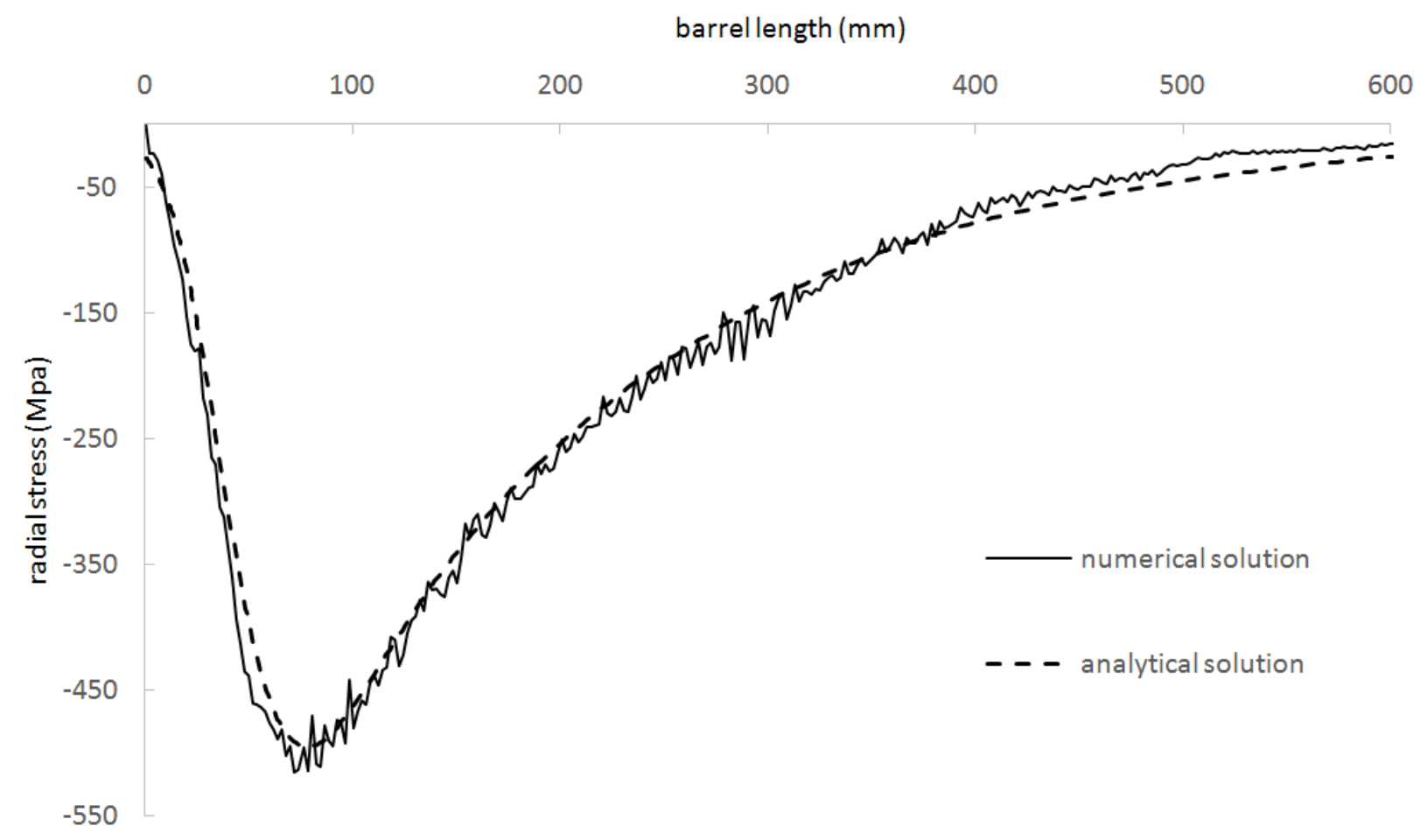

Figure 10. comparison between analytical and numerical solution for stress.

- In this paper, equilibrium equations of cylinder is considered as the closest model to gun barrel and a simple equation for radial expansion velocity is obtained considering boundary conditions. Expansion velocity can be calculated in every times entering boundary condition. The method used in this research is unique according to comparison analytical and numerical output, therefore it can be used in real situation, lab environment and testing experimental related to gunnery in order to reducing costs and timeconsuming.

- Basically choosing the sort of equation of state governing the explosive after explosion and material model is not affecting on the analytical technique but it is necessary to be chosen in such a way to conform to the terms and actual results. Hence, internal ballistic pressure equation is considered in general form, but it should be mentioned the equation has been extracted with very high precision software. But from the convergence of analysis and simulation results can be deduced the equation of state describes explosive products expansion behavior very well with the related coefficients. So the resultant can be used in calculation easily.

- Radial velocity in the analysis is verified according to comparison with simulation therefore other extractive parameters accuracy like radial and hoop stresses depended to radial velocity is sufficient.
- Generally it can be stated that analysis technique introduced in this paper is accurate and the application of material models in the process of expansion recall is complete according to the simulation results and a small amount of errors. So this model can be used to predict the results before testing experimental.

\section{References}

[1] Nadai, A., Theory of Flow and Fracture of Solid, Vol. 1, McGraw-Hill, New York, 1950, pp. 472-81.

[2] Davidson, T. E., et al., Overstrain of high-strength open-ended cylinders of intermediate diameter ratio, In Proceedings of the 1st International Congress on Experimental Mechanics, Pergamon Press, New York, 1963, pp. 335-52.

[3] Chen, P. C. T., A comparison of flow and deformation theories in a radially stressed annular plate. Trans. ASME, J. Appl. Mech., 40 (1973) 283-7

[4] Bland, D. R. "Elastoplastic thick-walled tubes of workhardening material subject to internal and external pressures and to temperature gradients."Journal of the Mechanics and Physics of Solids 4.4 (1956): 209-229.

[5] Odintsov, V. A., and V. V. Selivanov. "Behavior of a rigidly plastic cylindrical shell exposed to internal pressure." Journal of Applied Mechanics and Technical Physics 16.3 (1975): 457 460. 
[6] Xu Hong \&Chen Shuning, An analysis of an autofrettaged cylinder considering strain-hardening effect, Bauchinger effect and temperature dependence of material properties (Part 1 Analysis for open-ended cylinder), In Proceedings of the 6th International Conference on Pressure Vessel Technology, Vol. 1. Pergamon Press, Oxford, 1988, pp. 423-430.

[7] Liu, Yong. "Solution of an open-ended autofrettaged thickwalled cylinder by mixed BEM." J. Zhejang Institute of Technology 2 (1991): 55-60.

[8] Gao, Xin-lin. "An exact elasto-plastic solution for an openended thick-walled cylinder of a strain-hardening material." International journal of pressure vessels and piping 52.1 (1992): 129-144.

[9] Gao, X-L. "Elasto-plastic analysis of an internally pressurized thick-walled cylinder using a strain gradient plasticity theory." International journal of solids and structures 40.23 (2003): 6445-6455.

[10] Li, Mao-lin, and Ming-fu Fu. "Limit analysis of viscoplastic thick-walled cylinder and spherical shell under internal pressure using a strain gradient plasticity theory." Applied Mathematics and Mechanics 29 (2008): 1553-1559.

[11] Bagheri, Seyed Masood, "Introduce a new Model for Expansion Behavior of Thick-Walled Cylinder under Internal Explosive Loading with Numerical Analysis" Modares mechanical engineering, 15.3 (2015): 251-259

[12] Lu, W. Y. \& Hsu, Y. C. "Elastic-plastic analysis of a flat ring subjected to internal pressure" Acta Mech., 27 (1977) 155-72

[13] Lu An-qi, "Elastic-plastic analysis of stresses around a circular hole in an infinite sheet subjected to equal biaxial tension" Acta Mech. Solida Sinica, 5 (1984) 449-53
[14] Xu Hong \& Chen Shuning, "An analysis of an autofrettaged cylinder considering strain-hardening effect, Bauchinger effect and temperature dependence of material properties (Part 1 Analysis for open-ended cylinder)" In Proceedings of the 6th International Conference on Pressure Vessel Technology, Vol. 1. Pergamon Press, Oxford, 1988, pp. 423-430

[15] Liu Yong, "Solution of an open-ended autofrettaged thickwalled cylinder by mixed BEM" J. Zhejang Institute of Technology, (2) (1991) 55-60

[16] Vullo, V. "Circular Cylinders and Pressure Vessels Stress Analysis and Design"; Springer International Publishing: Switzerland, 2014.

[17] Paffumi, E.; Taylor, N. "Structural Response of a Large Pressure Vessel to Dynamic Loading"; JRC Sci.Tech. Rep., 2008.

[18] Yong, L.; Xin, W. L.; Wei, X. L.; Meng, Z. F. "Residual Stress Analysis of a Thick-Walled Cylinder in Dynamic Loading"; Int. J. Pres. Ves. Pip. (IJPVP), Vol. 60, 17-20, 1994

[19] Hojman, Sergio A. "A new conservation law constructed without using either Lagrangians or Hamiltonians." Journal of Physics A: Mathematical and General25.7 (1992): L291.

[20] Elliott, J. Richard, and Carl T. Lira. Introductory chemical engineering thermodynamics. Upper Saddle River, NJ: Prentice Hall PTR, 1999.

[21] Fenner,Roger T. Engineering elasticity. Halsted Press, 1986.

[22] Dobratz, Brigitta M. Properties of chemical explosives and explosive simulants. No. UCRL--51319; UCRL--51319 (REV. 1). comp. and ed.; California Univ., Livermore (USA). Lawrence Livermore Lab., 1972. 